\title{
Spatial reorientation of the carriage of a CNC lathe when reversing
}

\author{
Alexander Denisenko \\ Samara State Technical University, Samara, Russian Federation
}

\begin{abstract}
The process of machining on $\mathrm{CNC}$ machines of parts of a complex profile with multi-pass shaping schemes is accompanied by multiple changes in the direction of movement of the working bodies (reverse) during cutting and at idle. In this case, the formation of processing errors associated with the spatial reorientation of the carriage during reversal is possible, the cause of which is the mismatch of the vector of the traction force acting along the axis of the lead screw and the resulting resistance forces associated with cutting forces and friction in the guides. Based on the analysis of the forces acting on the carriage, a design and mathematical model is proposed for guides with prismatic and flat guides. Approbation of the proposed calculation method showed a good level of agreement between the calculated and experimental results. The regularities of the influence of the cutting forces, the location of the center of gravity of the support group, its mass and speed after the reverse on the value of the spatial reorientation have been established.
\end{abstract}

\section{Introduction}

The trend in the development of modern mechanical engineering is the constant tightening of requirements for the accuracy of products. The release of high-precision products in conditions of constantly increasing requirements for productivity, concentration of operations and multinationality in metalworking is possible only with the widespread use of metal-cutting equipment with CNC. In this regard, ensuring the high accuracy of CNC machines at the design stage makes it possible to guarantee the parametric reliability of the equipment.

The accuracy characteristics of the shaping movements of the machine are determined by the operation of two main subsystems: the main motion drive and the feed drives, the design and operation conditions of which are devoted to a significant number of modern studies [1-10].

Since CNC machines are most efficiently used when processing parts of a complex profile with multi-pass shaping schemes, the processing process is accompanied by multiple changes in the direction of movement of the working bodies (reverse) during cutting and at idle.

In this case, an error of the machine is formed associated with the loss of information during the reverse in the kinematic chain of the feed drive and the spatial reorientation of the support group. In the kinematic chain, the loss of information is associated with the presence of gaps in the chain of feed drives and elastic deformations determined by 
constant loads overcome by the drive. These losses form a positioning dead zone at position [11].

Much less attention is paid to the second component, due to the spatial reorientation of the support group during reverse [12,13], which requires a more detailed study of this phenomenon and its consideration in the design.

\section{Model of the spatial reorientation of the carriage when reversing}

Let us consider the phenomenon of spatial reorientation of the carriage during reverse on a lathe with prismatic front and flat back guides lying in the horizontal plane.

The main reason for the spatial reorientation of the lathe support group during reverse is the occurrence of a moment due to the mismatch of the traction force acting along the axis of the lead screw and the resultant resistance forces associated with cutting forces and friction in the guides.

In lathes with a horizontal plane of guides and the use of combined guides (flat and prismatic), the effect of this moment is significantly aggravated by the spatial turn of the part of the support group over the prismatic guide, which leads to elastic deformations of the carriage.

When compiling a design model of the spatial reorientation of the carriage during reverse, we will take into account the following assumptions:

- the carriage wings (parts of the carriage above each rail) are absolutely rigid, that is, they are not subjected to bending;

- we assume that for each point of the joint of the guides the same dependence of elastic deformations on pressure is valid as for flat joints;

- the mass of the support will be considered concentrated at two points: above the front guide $-m_{1}$ and over the back - $m_{2}$;

- contact deformations along the short side of the guide face are considered constant.

Consider the position of the part of the support over the prismatic guide. Taking into account contact processes, in the general case, this position is determined by the values of the clearances $\Lambda_{1} ; \Lambda_{2}$ and the values of the deformations $\Delta_{1} ; \Delta_{2}$ (at small moments causing the support to skew, the case of $\Lambda_{1}<0$ and $\Lambda_{2}<0$ is possible, that is, the absence of gaps) (Fig. 1).

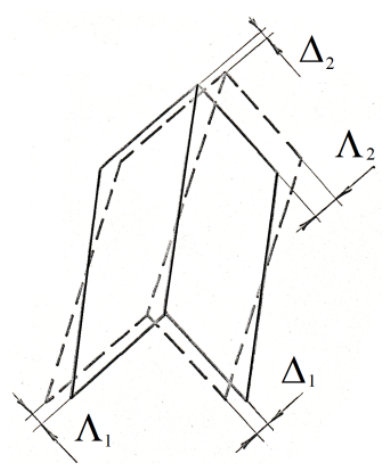

Fig. 1. Physical model of a part of the carriage above the prismatic guide with spatial reorientation.

Since in the general case the values are $\Lambda_{1} ; \Lambda_{2} ; \Delta_{1}$ and $\Delta_{2}$ are unequal in pairs, then, changing its spatial position, the front part of the support group (above the prismatic guide) causes deformation of the carriage bridge. 
Thus, the following forces and moments act on the caliper: $m_{1} g$ - weight of the front part of the support group; $m_{2} g$ - weight of the rear part of the support group; $Q$ - traction force on the lead screw; $Q l_{l s h}$ and $Q l_{l s v}$ - moments from traction force acting in the $\mathrm{XOZ}$ and YOZ planes, respectively; $Q_{h}$ and $Q_{v}$ - elastic forces arising from the deflection of the lead screw in the horizontal (XOZ) and vertical (YOZ) planes, respectively; $M_{e l}^{\prime}$ - the moment of elastic forces associated with torsional deformations of the carriage bridge (around the OX axis); $M_{e l}^{\prime \prime}$ - the moment of elastic forces associated with bending deformations of the carriage bridge (in the XOZ plane); $M_{l s h}$ and $M_{l s v}$ - moments of elasticity associated with the bending of the lead screw in the horizontal (XOZ) and vertical (XOZ) planes, respectively; $F_{f r}$ and $F_{f l}$ - frictional forces in the right and left sides of the front guide when moving along the $\mathrm{OZ}$ axis; $F_{f b}$ - frictional forces in the back guide when moving along the $\mathrm{OZ}$ axis; $R_{l}$ and $R_{r}$ - the resulting elastic contact deformations, respectively, in the left and right edges of the front guide; $R_{b}$ - the resulting elastic contact deformation of the back guide; $F_{l f}$ - hydrodynamic lifting force in the face of the front guide; $F_{l b}$ - hydrodynamic lifting force in the back guide.

Since the angle formed by the edges of the front guide is $90^{\circ}$, the skew of the carriage above the front guide can be viewed in two mutually perpendicular planes coinciding with the planes of the guide faces, in each of which the carriage rotates (Fig. 2).

\section{$\underline{\text { Left side }}$}

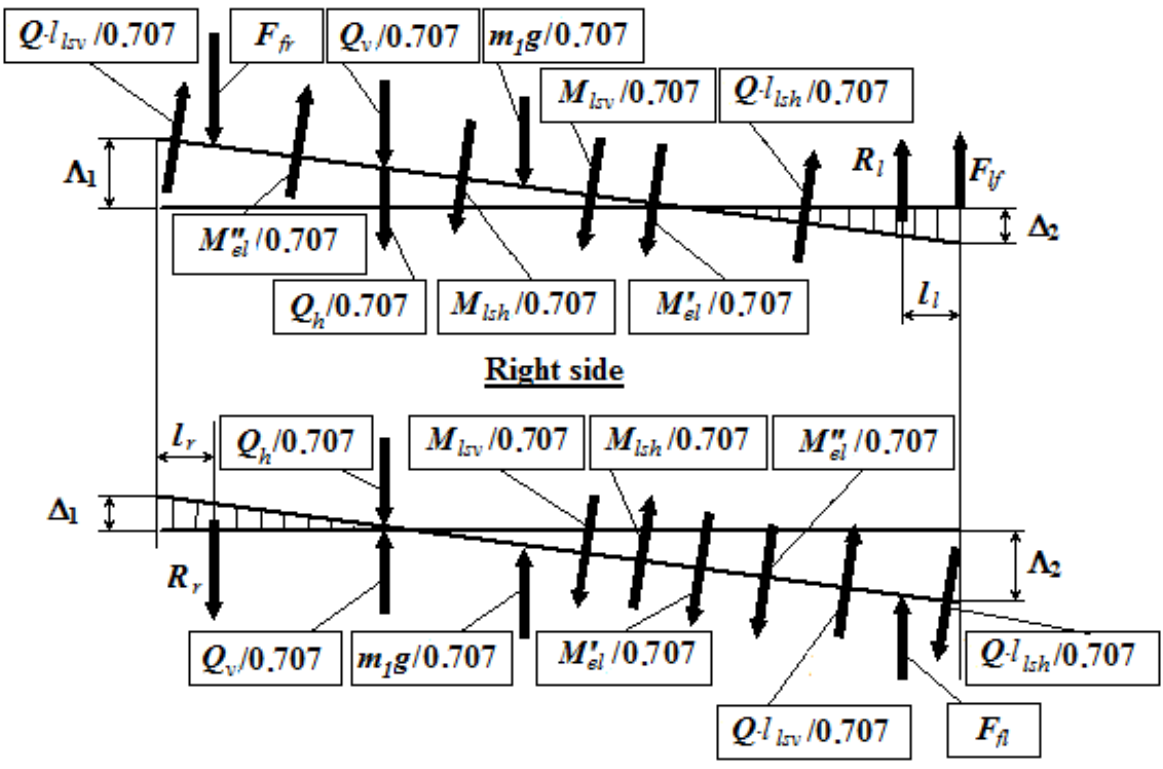

Fig. 2. Calculated model of the skew of the carriage over the prismatic guide.

Composing the equilibrium equations for the front and back parts of the carriage, we obtain a system of equations for determining its spatial position: 


$$
\left\{\begin{array}{l}
\sqrt{2}\left(R_{r}-F_{f r}+R_{l}-F_{f l}\right)-2 m_{1} g-2 Q_{v}+\sqrt{2} F_{l f}=0 ; \\
2 Q l_{l s v}-2 M_{e l}^{\prime}-2 M_{l s v}-2 Q_{v}\left(L-l_{1}-l_{m}^{\prime}\right)+\sqrt{2}\left(R_{r}-F_{f r}\right) \times \\
\quad \times\left(L-l_{m}^{\prime}-l_{r}\right)-\sqrt{2}\left(R_{l}-F_{f l}\right)\left(l_{m}^{\prime}-l_{l}\right)-\sqrt{2} F_{l f} l_{m}^{\prime}=0 ; \\
\quad \sqrt{2}\left(R_{l}+F_{f l}-R_{r}-F_{f r}\right)-2 Q_{h}+\sqrt{2} F_{l f}=0 \\
\\
2 M_{e l}^{\prime \prime}-2 M_{l s h}-\sqrt{2}\left(R_{r}+F_{f r}\right)\left(L-l_{m}^{\prime}-l_{r}\right)- \\
\quad-\sqrt{2}\left(R_{l}+F_{f l}\right)\left(l_{m}^{\prime}-l_{l}\right)+2 Q l_{l s h}-\sqrt{2} F_{l f} l_{m}^{\prime}=0 \\
Q-F_{f r}-F_{f l}-F_{f b}=0 \\
R_{b}=m_{2} g-F_{l b} \\
M_{e l}^{\prime}=R_{b}\left(l_{m}^{\prime \prime}-l_{b}\right)+F_{l b} l_{m}^{\prime \prime} \\
M_{e l}^{\prime \prime}=F_{f b} q
\end{array}\right.
$$

where parameters $L, l_{1}, l_{m}^{\prime}, l_{m}^{\prime \prime}, q, l_{l s h}$ and $l_{l s v}$ are shown in Fig. 3.

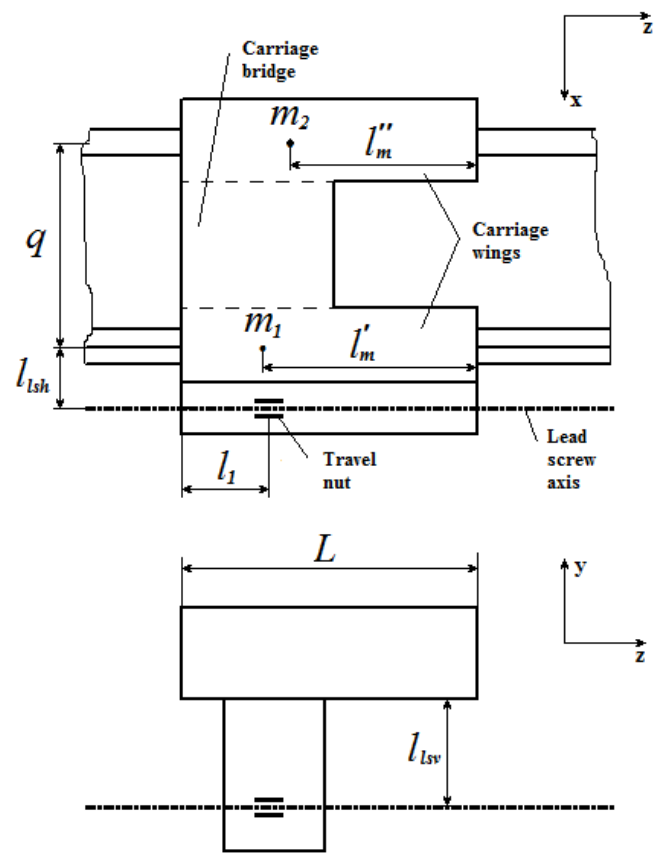

Fig. 3. Parameters of the calculation model

Numerical analysis of the computational model, the description of which is carried out by the system of equations (1), for the maximum values of the misalignments, measured experimentally on the machine, showed that the forces and moments of forces acting on the carriage when skewed differ by $1 . . .2$ orders of magnitude. In particular, the components associated with the elastic deformations of the lead screw, which is determined by its considerable length, are significantly smaller than others.

Based on the above, the system of equations (1) will take the form: 


$$
\left\{\begin{array}{l}
\sqrt{2}(1-f)\left(R_{r}+R_{l}\right)-2 m_{1} g+\sqrt{2} F_{l f}=0 ; \\
2\left(R_{r}+R_{l}+R_{b}\right) f l_{l s v}-2 R_{b}\left(l_{m}^{\prime \prime}-l_{b}\right)-2 F_{l b} l_{m}^{\prime \prime}-\sqrt{2} F_{l f} l_{m}^{\prime}+ \\
+\sqrt{2} R_{r}(1-f)\left(L-l_{m}^{\prime}-l_{r}\right)-\sqrt{2} R_{l}(1-f)\left(l_{m}^{\prime}-l_{l}\right)=0 ; \\
(1+f)\left(R_{l}-R_{r}\right)+F_{l f}=0 ; \\
2 R_{b} f q-\sqrt{2} R_{r}(1+f)\left(L-l_{m}^{\prime}-l_{r}\right)-\sqrt{2} F_{l f} l_{m}^{\prime}- \\
-\sqrt{2} R_{l}(1+f)\left(l_{m}^{\prime}-l_{l}\right)+2\left(R_{r}+R_{l}+R_{b}\right) f l_{l s h}=0 .
\end{array}\right.
$$

Coefficient of friction in guides

$$
f=f_{0}-\frac{\mu S_{H}}{N}\left[\frac{d_{H}^{2} f_{0}}{\varepsilon\left(h_{0}^{\prime}+d_{H}\right)^{3}}-\frac{1}{h_{0}^{\prime}}\right] V,
$$

where $f_{0}$ is the coefficient of static friction; $V$ - movement speed; $N$ - normal load; $\mu$ dynamic viscosity of the lubricant; $S_{H}$ - the area of the smallest of the rubbing surfaces; $d_{H}$ - average height of microroughness; $\varepsilon$ - the ratio of the height of microroughnesses to their length; $h_{0}^{\prime}$ is the average value of the minimum clearance between friction surfaces at $V=0$.

\section{Experimental research}

To check the correctness of the proposed calculation model of the spatial reorientation of the support group during reverse, the calculation method and the assumptions made in this case, experimental studies were carried out on lathes mod. 16B16F3. Non-contact inductive sensors recorded the change in the gap between the measuring plane of the sensor and the ground sections of the bed at the points shown in Fig. 4.

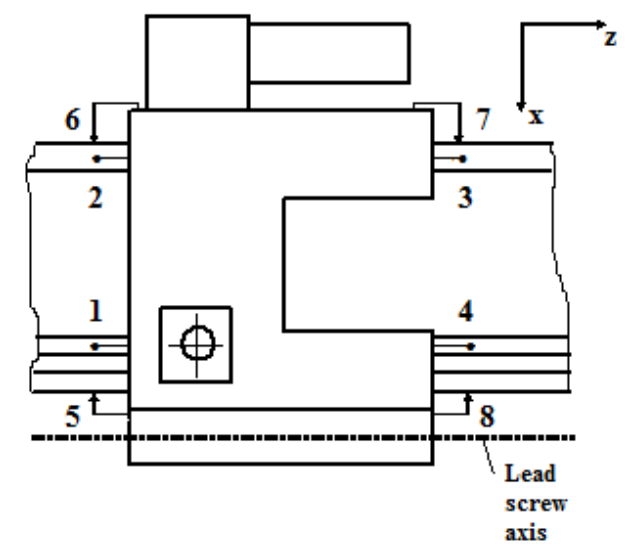

Fig. 4. The layout of the sensors when measuring the spatial reorientation of the support group during reverse.

Analysis of the process of skewing of the support group after the reverse indicates that the main part of its reorientation in space occurs approximately $0.2 \mathrm{~mm}$ after the reverse (Fig. 5). 


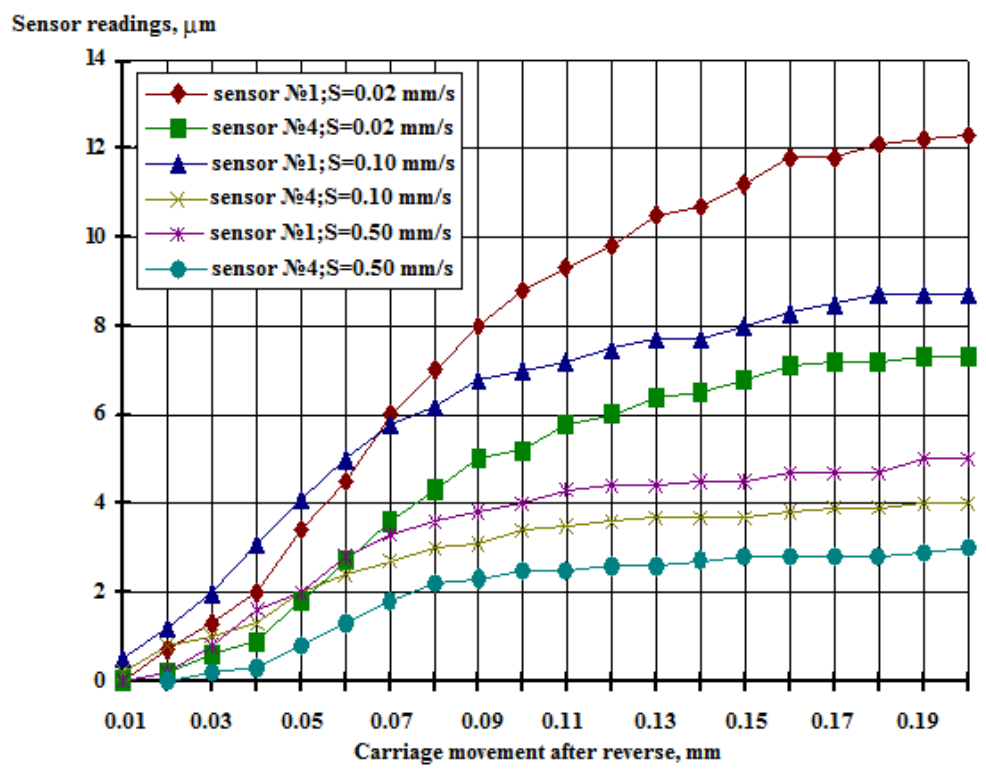

Fig. 5. Skew of the carriage at the points of installation of sensors 1 and 4 .

When the speed at which the reverse occurs, the following regularity is observed: with increasing speed, the lift of the front part of the carriage and the angle of its rotation around the axis OX, characterized by the difference between the readings of sensors 1 and 4 (Fig. 5), decrease. Comparison of the calculated and experimental skew curves depending on the speed at which the reverse occurs shows good qualitative and quantitative agreement (Fig. 6).

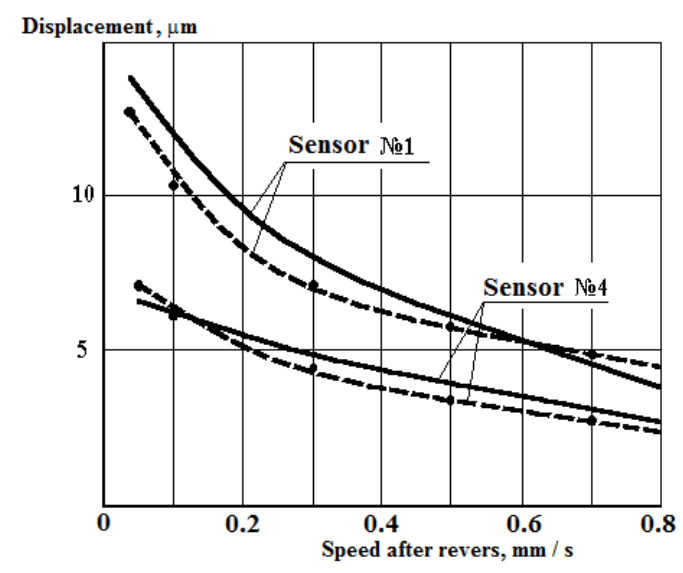

Fig. 6. Comparison of experimental (dotted line) and theoretical dependences of the skew at the points of installation of sensors 1 and 4 on the speed after reverse.

The skew value changes slightly with an increase in the mass of the support group without a practical shift in the center of gravity. The displacement of the center of gravity of the support group to the back guide leads to a redistribution of friction forces in the guides, and therefore increases the moment causing the skew. Evaluation of the effect of the cutting process on the spatial position of the support group was carried out when turning steel 45 for a pass through a cutter with a replaceable carbide triangular plate $\left(\gamma=12^{\circ} ; \alpha=\right.$ 
$7^{\circ} 30^{\prime}$ ) at low feeds ( $S=0.1$ and $0.5 \mathrm{~mm} / \mathrm{s}$ ) at different spindle speeds. The depth of cut varied within $0.5 \ldots 3.5 \mathrm{~mm}$. The experiment was carried out according to the following scheme: the caliper moved in the $+\mathrm{Z}$ direction, then there was a reverse and movement in the $-Z$ direction. Then the cutter was cut into the workpiece and its further turning. The distance from the reversal point to the infeed was determined from the condition that during this time the skew at idle speed reached almost the maximum value. In all the experiments carried out, when turning per pass, at the moment of cutting the cutter into the workpiece, an increase in the skew occurred. Moreover, with an increase in the depth of cut, no regular increase in skew was observed. For example, the magnitude of the increase in skew during plunging for points 1 and 4 (Fig. 4) at a spindle speed of $450 \mathrm{rpm}$ was $1 \ldots 3$ and $0.3 \ldots 0.5$ $\mu \mathrm{m}$, respectively, in the entire range of cutting depths.

\section{Conclusions}

As a result of the mismatch of the vector of the resultant of the friction forces in the guides with the stiffness axis of the support group during the reverse movement, its spatial reorientation occurs, the value of which decreases with increasing speed after the reverse.

The spatial position of the support group with a prismatic front and flat back guides lying in the horizontal plane, when reversed, is determined by the position of the carriage above the front guide.

The displacement of the center of gravity of the support group to the back guide causes an increase in the skew.

The forces acting on the carriage of a lathe during reorientation are considered, and a calculation and mathematical model is proposed that determine its bias during reverse.

Experiments carried out during cutting showed an increase in skew at the moment the cutter plunges into the workpiece.

Comparison of the calculated and experimental skew curves depending on the speed after reversing showed good qualitative and quantitative agreement.

\section{References}

1. J.P. Choi, S.J. Lee, H.D. Kwon, Roundness error prediction with a volumetric error model including spindle error motions of a machine tool, Advanced Manufacturing Technology, 21, pp. 923-928 (2003)

2. A. Denisenko, Impact of manufacturing errors of ball screw system on performance characteristics, MATEC Web of Conferences 224, 01042 (2018), https://doi.org/10.1051/matecconf/201822401042

3. V.G. Gusev, N.V. Malova, Schemes of processing spherical surfaces on multifunctional machines with CNC, Innovative technologies in automated mechanical engineering and valve engineering, , pp. 60-63 (2010)

4. V. Gagnol, B.C. Bouzgarrou, P. Ray, C. Barra, Stability-Based Spindle Design Optimization, Journal of Manufacturing Science and Engineering, v. 129, Issue2, pp. 407-415 (2006)

5. M. Soshi, S. Yu, S. Ishii, K. Yamazaki, Development of a high torque-high power spindle system equipped with a synchronous motor for high performance cutting, CIRP Annals - Manufacturing Technology, v. 60, Issue 1, pp. 399-402 (2011)

6. V.V. Kozyrev, M.V. Kozhevnikova, System for analysis and synthesis of the power part of mechatronic modules, Mechatronics, automation, control, v. 2, pp. 28-32 (2009) 
7. V.V. Bushuev, V.A. Kuzovkin, V.V. Molodtsov, V.V. Filatov, Analysis of errors of the metal cutting machine feed drive and methods of reducing their, Measuring technology, 6, pp. 18-22 (2006)

8. A.A. Seregin, A.G. Kravtsov, Monitoring of technological systems when processing precise surfaces of difficult contour,Bulletin of the South Ural State University. Series: Mechanical Engineering, v. 18 (4), pp. 48-56 (2018)

9. E. Abele, Y. Altintas, C. Brecher, Machine tool spindle units, CIRP Annals Manufacturing Technology, v. 59, Issue 2, pp. 781-802 (2010)

10. A.F. Denisenko, Development of drive designs for metal-cutting machine tools with isotropic coordinate compliance, Bulletin of Samara State Technical University. Ser. "Technical science", 2 (30), pp. 119-125 (2011)

11. A. Denisenko, Prediction of the insensitivity zone in the design of the feed drive CNC lathe, IOP Conf. Series: Materials Science and Engineering, 709, 022058, (2020) doi:10.1088/1757-899X/709/2/022058

12. N.V. Emelyanov, V.L. Zubenko, I. V. Emelyanova, CAD, CAE design technologies taking into account friction forces of moving joints of units of $\mathrm{CNC}$ lathes, Bulletin of the Samara Scientific Center of the Russian Academy of Sciences, v. 18, No. 4 (2), pp. 279-284, (2016)

13. A.F. Denisenko, The spatial position of the translationally moving unit of a metalcutting machine taking into account the hydrodynamic lifting force acting at low speeds, Bulletin of the Samara State Technical University. Ser. "Technical science". 15, pp. 119-123, (2002) 